\title{
The prevalence of adult de novo scoliosis: A systematic review and meta-analysis
}

\author{
Jeb McAviney ${ }^{1} \cdot$ Carrie Roberts $^{2} \cdot$ Bryony Sullivan $^{2} \cdot$ Alexander J. Alevras $^{2} \cdot$ Petra L. Graham $^{3}$ (D) \\ Benjamin Thomas Brown ${ }^{1,2}$ (1)
}

Received: 17 November 2019 / Revised: 28 April 2020 / Accepted: 6 May 2020 / Published online: 22 May 2020

(c) The Author(s) 2020

\begin{abstract}
Introduction Primary degenerative scoliosis represents a new scoliosis developing in patients with no prior history of spinal curvature. Researchers sought to determine the prevalence of this type of scoliosis.

Methods MEDLINE, Embase, CINAHL, Web of Science and PubMed were searched from inception to 28th March, 2018. Studies that assessed adults from the general population for scoliosis using imaging techniques were included. Studies were included only if the study authors had excluded participants with previously diagnosed scoliosis and/or spinal disorders. Mixed-effects logistic-regression was used to establish an overall prevalence estimate with $95 \%$ confidence intervals (primary outcome) and to examine the effect of age and sex (secondary outcomes).

Results Four cross-sectional studies and one cohort study, involving 4069 participants (66.6\% Female), aged between 41 and 94 years, were eligible for inclusion. Reported prevalence figures ranged from 13 to $68 \%$. The pooled prevalence estimate from the mixed-effects logistic regression analysis was 37.6\% (95\% CI 18.7-61.8). Females were more likely to suffer from scoliosis compared with males ( $p<0.001$ ), with prevalence figures of $41.2 \%$ (95\% CI 20.7-65.8) versus $27.5 \%$ (95\% CI 12.2-51.1), respectively. Individuals aged $<60$ years had a prevalence of $13 \%$ (95\% CI 5.2-30.2), whereas the prevalence estimates were substantially higher in the $>60$ age group [36\% (95\% CI 17.4-60.6)].

Conclusion Primary degenerative scoliosis is a highly prevalent condition, especially in females. Further research targeting this type of scoliosis is required to obtain more precise global prevalence estimates and to understand the influence of age and sex.
\end{abstract}

Keywords Scoliosis $\cdot$ Prevalence $\cdot$ Adult

$\begin{array}{ll}\text { Abbreviations } \\ \text { AXIS } & \text { Appraisal tool for cross-sectional studies } \\ \text { DEXA } & \text { Dual-energy X-ray absorptiometry } \\ \text { LAT } & \text { Lateral } \\ \text { MeSH } & \text { Medical subject headings } \\ \text { PA } & \text { Posteroanterior }\end{array}$

Benjamin Thomas Brown

benjamin.brown@mq.edu.au

1 Sydney Scoliosis Clinic, Kirk Place, Level 5, Suite 5.08, 15 Kensington St, Kogarah, NSW 2217, Australia

2 Faculty of Science and Engineering, Macquarie University, Balaclava Rd, North Ryde, Macquarie Park, NSW 2109, Australia

3 Centre for Economic Impacts of Genomic Medicine (GenIMPACT), Macquarie Business School, Macquarie University, Macquarie Park, NSW 2109, Australia
PDS Primary (de novo) degenerative scoliosis

STROBE Strengthening the reporting of observational studies in epidemiology

\section{Introduction}

Scoliosis is 3-dimensional spinal deformity that is diagnosed by measuring the magnitude of the spinal deviation on standing coronal plane radiographs [1]. The threshold for diagnosis is typically defined as a Cobb angle measurement of $\geq 10^{\circ}$ [2]. There are many different types of scoliosis that may present at different points across the lifespan. With respect to adult populations, Abei [3] described three main types of scoliosis: type I-primary (de novo) degenerative scoliosis; type II-progressive idiopathic scoliosis in an adult patient; or type III-(a) secondary degenerative scoliosis in response to idiopathic or other forms of scoliosis 
or primary deformity/anomaly located in the spine, trunk or extremities or (b) any systemic condition that may affect bone metabolism (e.g. osteoporosis) combined with asymmetrical arthritis or vertebral fracture. Two additional types, described by Berven and Lowe [4] in the Scoliosis Research Society classification for adult spinal deformity document, include post-traumatic and post-surgical scoliosis. Of the various types described above, primary (de novo) degenerative scoliosis and secondary degenerative scoliosis are most common in adult patients who present clinically [3]. Primary (de novo) degenerative scoliosis (PDS) is the focus of this review. Primary degenerative scoliosis represents a new (de novo) curve that develops in patients with no prior history of scoliosis, and typically affects the lumbar or thoroacolumbar spine. Patients with PDS typically present with progressive postural deformity accompanied by axial back pain [5]. In more severe cases, patients may experience radiculopathy, myelopathy and/or neurogenic claudication [6, 7]. The signs and symptoms in PDS patients are driven by asymmetrical disc degeneration, spondylosis/facet incompetence, asymmetrical changes in the lumbar paraspinal muscles [8], and hypertrophy and calcification of the ligamentum flavum [6]. Changes in the structure, function, and physiological alignment of the spine lead to asymmetrical loading which provokes further degenerative change, accelerated curve progression, muscle changes [9], and postural collapse. Alterations in the sagittal spinal parameters also contribute to coronal plane deviation. The hallmark radiological features of PDS are deviations from normal spinal alignment that include abnormal, rigid/inflexible, spinal curvatures typically located in the lumbar or thoracolumbar spine, a decrease in the normal lumbar lordosis, varying degrees of -olisthesis in the transverse, coronal and/or sagittal planes, and spinal stenosis $[4,6,7,10]$.

It is estimated that by 2050 , the proportion of the world's population aged $>60$ years will nearly double [11], which will place significant strain on healthcare systems throughout the world. The prevalence of PDS is known to increase with age, with progressive spondylosis, sedentary behaviours, sarcopenia and decreases in bone mineral density contributing to this trend $[7,12,13]$. The reported prevalence estimates for PDS are quite varied ranging from 2 [14] to $68 \%$ [15]. Accurate prevalence statistics are important for clinicians, epidemiologists and policy makers, and given that PDS is strongly associated with ageing, it is vital that the prevalence of this condition be more precisely mapped. The aim of this study was to determine the pooled prevalence of PDS based on reports from the existing literature and to determine the influence of age and sex.

\section{Methods}

The authors sought to retrieve all observational studies that reported on the prevalence of scoliosis in samples of adult participants ( $\geq 18$ years of age) taken from either a general population or primary care setting. To be eligible, studies had to have utilized medical imaging that allowed for the radiographic diagnosis of scoliosis (e.g. X-ray), and that any radiographic interpretation had to have been performed by a suitably trained clinician. The threshold for diagnosis of scoliosis in this review was defined as a coronal plane Cobb angle measurement of $\geq 10^{\circ}$. In order to obtain prevalence figures that would be most representative of adults with de novo degenerative scoliosis, studies were included only if the authors had explicit exclusion criteria relating to participants with previously diagnosed scoliosis or spinal disorders or a history of spinal surgery. Studies written in either English, Dutch, Greek or German that had been published in full-text, in peer-reviewed journals were included. There were no publication date restrictions imposed. Studies involving participants with types of scoliosis other than primary degenerative scoliosis were excluded.

A search of the MEDLINE, Embase, CINAHL, Web of Science and PubMed databases was performed from database inception to the 28th of March 2018. A combination of search terms, including medical subject headings (MeSH), pertaining to adults with scoliosis (adult spinal deformity, de novo degenerative scoliosis, adult degenerative scoliosis, primary degenerative scoliosis) were used. An example of the search strategy for the PubMed database is provided in "Appendix". The search results from the five databases were imported into reference manager software and all duplicates were removed. A preliminary screen, based on title and abstract only, was then performed by two authors. The full texts of the remaining studies were then retrieved, and the eligibility criteria were applied independently by two authors. Any disagreements were settled by consensus. Forward and reverse citation tracking, based on the list of eligible studies, was performed to identify relevant studies that had not been captured by the primary search strategy. No gray literature was included in this study.

Data were then extracted independently by two authors using a customized template which was piloted prior to use. Information was extracted regarding: study design; ethics approval; study setting; sampling methods; recruitment dates; eligibility criteria; outcome measures; method/s of diagnosis; response rates; participant characteristics; scoliosis parameters; and prevalence statistics; and other study findings. Three research groups were contacted as important data were not contained in the original reports; however no return correspondence was received in each of the three cases. 
A risk of bias and a reporting quality assessment were conducted for each eligible study using the appraisal tool for cross-sectional studies (AXIS) [16] and the strengthening the reporting of observational studies in epidemiology (STROBE) checklist [17], respectively. For the risk of bias assessment, a rating of either high, low or unclear was given based on recommendations from the Cochrane Collaboration [18]. All data were then collated and summarized as the proportion of participants diagnosed with scoliosis (prevalence). Mixed-effects logistic-regression was used to establish an overall prevalence estimate with $95 \%$ confidence intervals, and to examine the effect of age and sex on prevalence. The mixed-effects model controlled for multiple groups from the same study (from sex and age groupings for example) via a random intercept. A 5\% significance level was set for all analyses. The analysis was conducted using the R statistical software program version 3.4.1 (R Core Team, Vienna, Austria). All studies were included in the final analysis and there were no sensitivity analyses planned.

\section{Results}

The primary search strategy identified 1667 publications. Of these, 395 were flagged as duplicates and were removed. The remaining 1272 publications were then screened by title and abstract. One hundred and one publications were deemed suitable for full-text appraisal. The full-text of these publications was obtained and the eligibility criteria were applied independently by two authors. Five publications [15, 19-22], detailing the results from five unique studies, satisfied the criteria for inclusion. Forward and reverse citation tracking, based on the five eligible publications, returned a further 438 items. These publications were screened by title and abstract; however none satisfied the eligibility criteria. Data from the eligible studies were extracted, collated and analysed. See Fig. 1 for a full description of the search and selection process.

The details of each study are summarized in Table 1. There were four cross-sectional studies and one cohort study. There were 4069 participants included in total. The largest study involved 2395 participants and the smallest enrolled only 75 participants. The overall age range for the participants in the included studies was 40-90 years. The majority of participants $(66.6 \%)$ were female, and all but one study recruited both males and females. The earliest recruitment period reported was 1992, and the most recent was 2012. Three of the five studies recruited participants involved with health promotion programs, one study recruited from a population register, and one study recruited participants from the local community via newspaper advertisements. All participants enrolled in each of the individual research studies were volunteers. Individuals who had previously been diagnosed with scoliosis or spinal deformity were excluded in all studies. The diagnosis of scoliosis was made via coronal plane Cobb angle measurements (threshold $\geq 10^{\circ}$ ) based on standing, plain films in the majority of studies $[15,19$, 21, 22] or dual-energy X-ray absorptiometry [DEXA] in one study [20]. The most common curve locations reported were in the lumbar and thoracolumbar regions. Other variables that were recorded were: pain rating scales $[15,22]$; radiographic parameters [15, 19-22]; quality of life [15], medical [22] or haematological nutritional analysis [15]; anthropometric measures [20]; and demographics [15, 19-22]. All five studies were published in English and were conducted in either in the Americas [15] or Asia [19-22].

Prevalence ranged from 13.3 to $68 \%$ in the included studies. The number of cases and total number of participants from each study were then combined using mixed-effects logistic regression analysis, which produced a pooled prevalence estimate of $37.6 \%$ (95\% CI 18.7-61.8). Only some studies included a sufficiently detailed breakdown of prevalence rates based on age. The data presented allowed two age groups to be extracted: $<60$ and $>60$ years of age. The analysis revealed that individuals aged $<60$ years had a prevalence of $13.2 \%$ (95\% CI 5.2-30.2), whereas the prevalence estimates were significantly higher in the $>60$ age group (35.9\% [95\% CI 17.4-60.6]; $p<0.001$ ). When looking at sex as a predictor, females were significantly more likely to suffer from scoliosis compared to males $(p<0.001)$, with a prevalence estimate of $41.2 \%$ (95\% CI 20.7-65.8) versus $27.5 \%$ (95\% CI 12.3-51.2), respectively.

A subgroup analysis of the studies from the Asiatic region only revealed a pooled prevalence estimate of $30.8 \%(95 \%$ CI 14.9-53.5). The differences in prevalence rates between the sexes [females $33.8 \%$ (95\% CI 16.6-57) and males $21.7 \%$ (95\% CI 9.7-42.1)] and age groups [<60 years $10.0 \%(95 \%$ CI 4.1-22.5), and > 60 years $28.9 \%$ (95\% CI 14.0-50.7)] remained significant.

There was considerable heterogeneity in the included studies. There were large differences in sample sizes between studies, and also in the reported prevalence rates, which prohibited the use of some meta-analytic techniques. More granular data regarding age and sex could not be obtained, despite several attempts to precure this additional information from the authors of the original reports.

The details of the risk of bias assessment are detailed in Table 2. The risk of bias assessment revealed that sample size calculations were not performed in any of the included studies. Non-responders were not described, categorized or enumerated in any of the included studies. More than half $[15,19,21]$ of studies did not include sufficient detail regarding their statistical methods. The majority $[15,19$, 21] of studies did not adequately describe how the limitations of their study may have affected their findings. Overall however, the risk of bias was judged by review authors to 
Fig. 1 Flowchart of the search and selection process
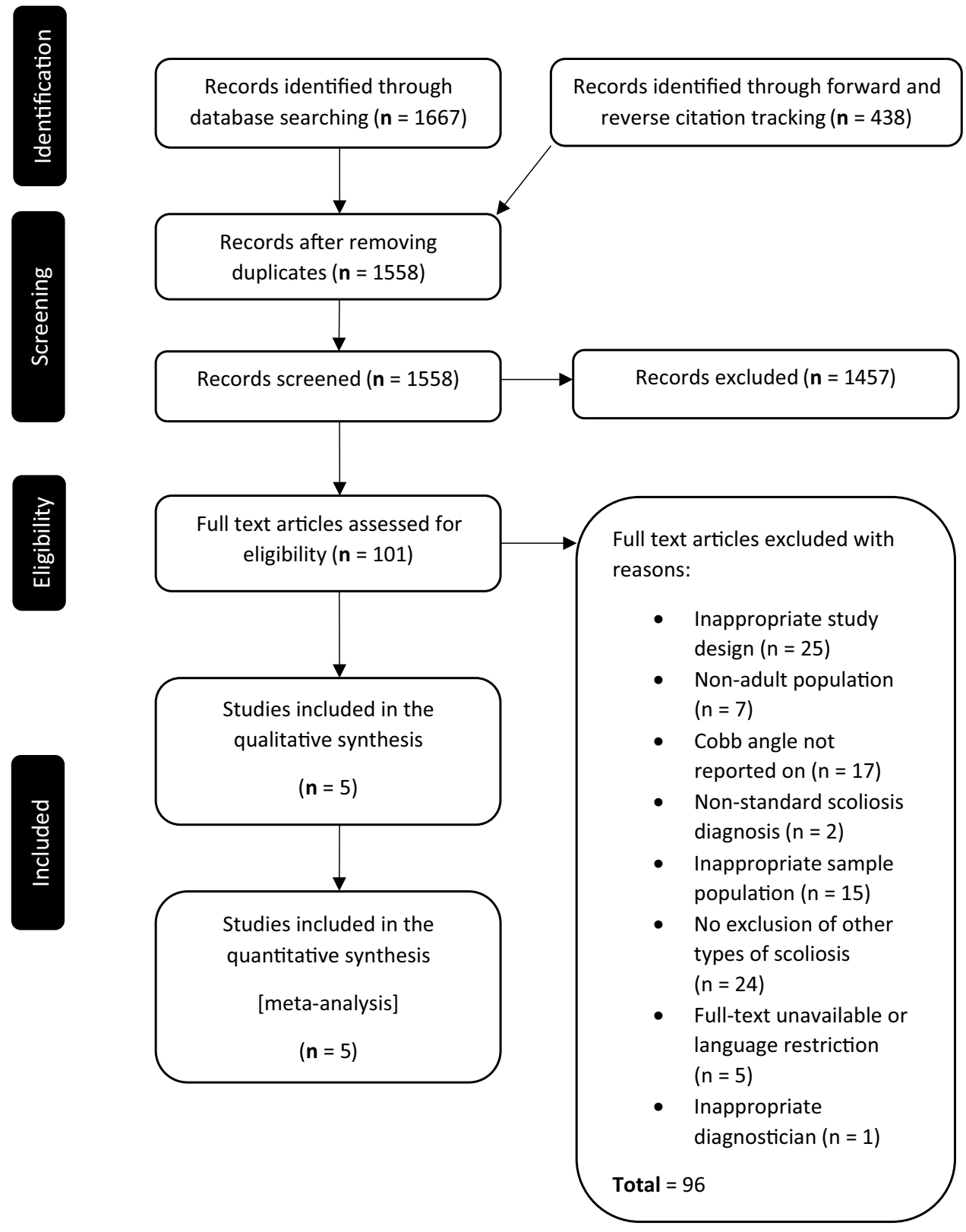

be low in all of the included studies and the methodological short-comings were deemed unlikely to bias the reported prevalence estimates significantly.

Similar findings were reflected in the reporting quality assessment detailed in Table 3. With respect to the crosssectional studies, in all studies, authors did not define the study design in the study title. The authors of the various cross-sectional studies did not describe how sample sizes were calculated or provide a description of attempts to minimize bias. The numbers of individuals at each stage of the study was not described and 95\% confidence intervals for prevalence estimates were also not provided in any of the studies. With reference to the single cohort study [21], the authors failed to provide adequate information on sample size calculation, statistical methods, numbers of participants at each stage of the study and/or associated missing data. The direction and magnitude of the effect of any potential bias was also not addressed in the cohort study.

\section{Discussion}

We have used the available evidence to establish an estimate of the prevalence of PDS. The findings of this review estimate the prevalence of this condition to be $38 \%$ for adults aged between 40 and 90 years. There is a significantly higher prevalence of PDS in females, and individuals aged $>60$ years have a significantly higher prevalence 


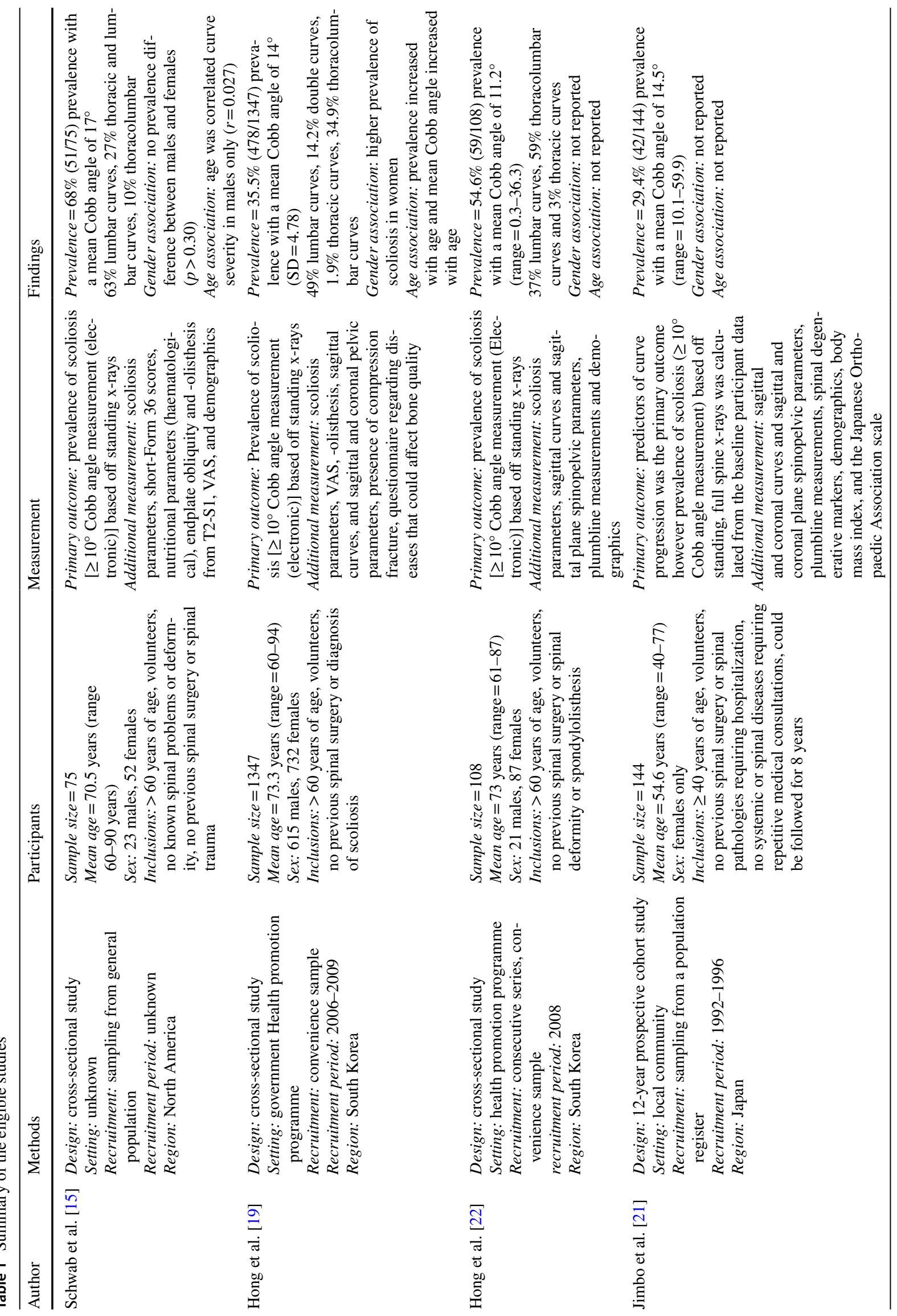




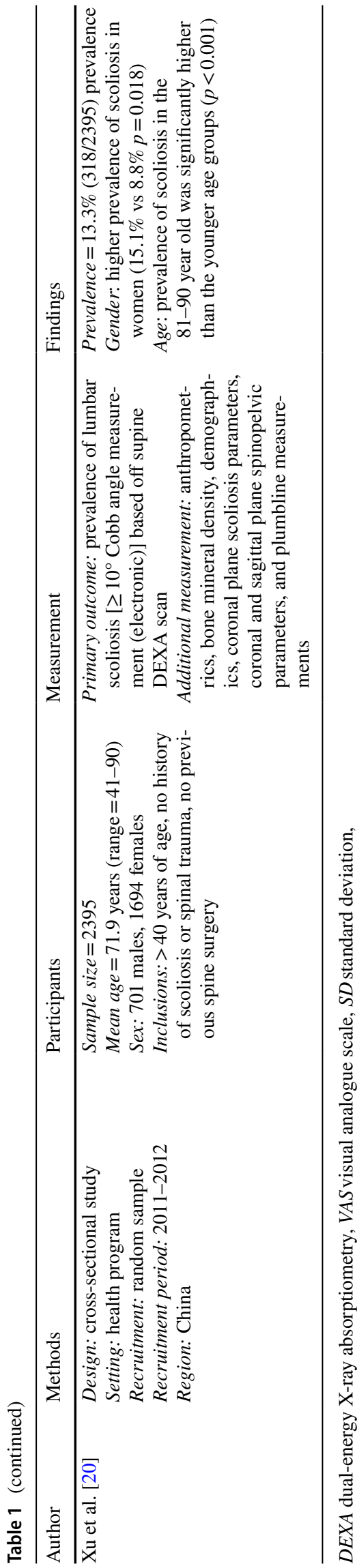

of PDS compared to those aged 40-60 years. The overall quality of the studies included in this review was high. Although, each of the eligible studies could have been strengthened if the associated researchers had presented more information regarding both respondents and nonrespondents, and included details of a priori sample size calculations. One of the difficulties in calculating sample size for a cross-sectional study is that an estimate of the expected prevalence in the population of interest must be included in the calculation. The prevalence estimate can be derived from pilot studies or from previous reports [23]. Given the general paucity of good quality prevalence studies, (and studies in general) in this area, and the wide-ranging prevalence figures that do exist, estimating prevalence for sample size calculations is not a straightforward task. It is not known to what extent the methodological flaws in the included studies may have influenced the prevalence estimates presented in this review.

With respect to the influence of age on prevalence rates, the findings of this review are in alignment with the existing literature on PDS and with the basic science research into spinal degeneration. Normal ageing is accompanied by degenerative change in the spine [13, 24, 25]. Asymmetrical spondylotic-change creates asymmetrical loading which drives a vicious cycle of degeneration and frustrated healing, especially in the lower spine $[25,26]$. These changes are strongly implicated in the development and progression of PDS. It therefore follows logically that aging would be associated with an increased prevalence of PDS, and a higher prevalence of lumbar and thoracolumbar scoliosis. With regards to the influence of sex on PDS, the picture is less clear. Sex influences on rates of lumbar spondylosis in adults are varied and may be influenced by ethnicity [27-31]. Moreover, the decreases in bone mineral density that occur in post-menopausal women also do not fully explain the higher prevalence of PDS in females, with some researchers highlighting increases in spinal bone mineral density in patients with degenerative scoliosis [32]. Complex interactions between conditions such as osteoporosis, osteoarthritis, disc degeneration, and joint and/or ligamentous laxity, for which there is a strong female predilection [33], may be contributing to the higher prevalence of PDS witnessed in this group. Further research is required to determine the exact role of sex in the pathogenesis and progression of PDS.

This review provides a more robust estimate of PDS prevalence which will aid clinicians in determining the likelihood of PDS in their patients. This will directly influence clinical-decision-making and may alter the choice of physical examination procedures and influence the rate of requests for paraclinical information (i.e. imaging) in adults presenting with scoliosis [34]. By providing a more accurate measure of PDS in the population governments and policy makers can more effectively plan and allocate resources for 
Table 2 Results from the risk of bias assessment based on the AXIS tool

\begin{tabular}{lllllllllllllllllllll}
\hline Study & Q1 & Q2 & Q3 & Q4 & Q5 & Q6 & Q7 & Q8 & Q9 & Q10 & Q11 & Q12 & Q13 & Q14 & Q15 & Q16 & Q17 & Q18 & Q19 & Q20 \\
\hline Schwab et al. [15] & + & + & $\mathrm{X}$ & + & + & + & $\mathrm{X}$ & + & + & $\mathrm{X}$ & $\mathrm{X}$ & + & $\mathrm{X}$ & $\mathrm{X}$ & + & $\mathrm{X}$ & + & $\mathrm{X}$ & + & + \\
Hong et al. [19] & + & + & $\mathrm{X}$ & + & + & + & $\mathrm{X}$ & + & + & + & + & + & $\mathrm{X}$ & $\mathrm{X}$ & + & + & + & + & + & + \\
Hong et al. [22] & + & + & $\mathrm{X}$ & + & + & + & $\mathrm{X}$ & + & + & + & $\mathrm{X}$ & + & $\mathrm{X}$ & $\mathrm{X}$ & + & + & + & $\mathrm{X}$ & + & $\mathrm{X}$ \\
Jimbo et al. [21] & + & + & $\mathrm{X}$ & $\mathrm{X}$ & + & + & $\mathrm{X}$ & + & + & + & $\mathrm{X}$ & + & $\mathrm{X}$ & $\mathrm{X}$ & + & + & + & $\mathrm{X}$ & + & + \\
Xu et al. [20] & + & + & $\mathrm{X}$ & + & + & + & $\mathrm{X}$ & + & + & + & + & + & $\mathrm{X}$ & $\mathrm{X}$ & + & + & + & + & + & + \\
\hline
\end{tabular}

$+=$ The authors adequately addressed this potential source of bias, $\mathrm{X}=$ The authors did not adequately address this potential source of bias

The order of the responses for Q19 has been reversed for all studies to highlight that an answer in the negative reduces the risk of bias

Table 3 Quality of reporting assessment based on the STROBE guidelines

\begin{tabular}{|c|c|c|c|c|c|c|}
\hline $\begin{array}{l}\text { Study } \\
\text { (Year) }\end{array}$ & $\begin{array}{c}\text { Hong et al. } \\
{[19]} \\
\text { (cross-sectional) }\end{array}$ & $\begin{array}{c}\text { Hong et al. } \\
{[22]} \\
\text { (cross-sectional) }\end{array}$ & $\begin{array}{c}\text { Xu et al. } \\
{[20]} \\
\text { (cross-sectional) }\end{array}$ & $\begin{array}{c}\text { Schwab et al. } \\
{[15]} \\
\text { (cross-sectional) }\end{array}$ & $\begin{array}{l}\text { Study } \\
\text { (Year) }\end{array}$ & $\begin{array}{c}\text { Jimbo et al. } \\
\text { [21] } \\
\text { (cohort) }\end{array}$ \\
\hline Question & & & & & Question & \\
\hline $1 \mathrm{~A}$ & $x$ & $x$ & $x$ & $x$ & Q1A & + \\
\hline $1 \mathrm{~B}$ & + & + & + & + & Q1B & + \\
\hline Q2 & + & + & + & + & Q2 & + \\
\hline Q3 & + & + & + & + & Q3 & + \\
\hline Q4 & + & + & + & + & Q4 & + \\
\hline Q5 & + & + & + & $x$ & Q5 & + \\
\hline Q6 & + & + & + & + & Q6A & + \\
\hline Q7 & + & + & + & + & Q6B & \\
\hline Q8 & + & + & + & + & Q7 & + \\
\hline Q9 & $x$ & $x$ & $x$ & $x$ & Q8 & + \\
\hline Q10 & $x$ & $x$ & $x$ & $x$ & Q9 & + \\
\hline Q11 & + & + & + & + & Q10 & $x$ \\
\hline Q12A & + & + & + & $x$ & Q11 & + \\
\hline Q12B & $x$ & + & + & $x$ & Q12A & $x$ \\
\hline Q12C & & & & & Q12B & + \\
\hline Q12D & & & & & Q12C & \\
\hline Q12E & & & & & Q12D & $x$ \\
\hline Q13A & + & + & $x$ & $X$ & Q12E & $x$ \\
\hline Q13B & & & & & Q13A & $x$ \\
\hline Q13C & $x$ & $x$ & $x$ & $x$ & Q13B & $x$ \\
\hline Q14A & + & + & + & + & Q13C & + \\
\hline Q14B & & & & & Q14A & + \\
\hline Q15 & + & + & + & + & Q14B & $x$ \\
\hline Q16A & $x$ & $x$ & $x$ & $x$ & Q14C & + \\
\hline Q16B & + & + & + & + & Q15 & + \\
\hline Q16C & & & & & Q16A & + \\
\hline Q17 & + & + & + & + & Q16B & + \\
\hline Q18 & + & + & + & + & Q16C & $x$ \\
\hline Q19 & + & + & + & + & Q17 & + \\
\hline Q20 & + & + & + & + & Q18 & + \\
\hline Q21 & + & + & + & + & Q19 & $x$ \\
\hline \multirow{3}{*}{ Q $\angle 2$} & & & + & $X$ & Q20 & + \\
\hline & & & & & Q21 & + \\
\hline & & & & & Q22 & \\
\hline
\end{tabular}

Cross-sectional studies (Left), Cohort study $($ Right $),+=$ Authors adequately addressed this aspect of reporting, $\mathrm{X}=$ Authors did not adequately address this aspect of reporting. Shaded areas represent aspects of reporting that were not applicable to the study and/or could not be assessed by the reviewers 
the development and delivery of health services and public health initiatives for this group [35].

There are some limitations associated with this review. One of the main difficulties for researchers seeking to establish a prevalence estimate for the different types of adult scoliosis is that in any community-based sample there will be a vast array of different aetiologies for the spinal curvatures found in respondents. This type of scenario is of course a challenge faced in all types of research, but it is particularly difficult when researching adult scoliosis. Recruiting subjects with PDS requires that the explicit and exhaustive exclusion of other variants of scoliosis be stated in the eligibility criteria. While we included studies that explicitly tried to exclude subjects with other types of scoliosis, it is possible that some non-PDS participants were still present. For example, mild idiopathic adolescent scoliosis can often go unnoticed due to minimal aesthetic impact [14]. These types of cases may not present clinically until later in life which would serve to inflate the prevalence estimates of PDS. Moreover, in late stage presentations, it can be very difficult to discern between a secondary degenerative idiopathic scoliosis patient and a PDS case [7]. Precise prevalence rates of PDS will remain elusive unless researchers can incorporate access to historical medical records and thorough pre-screening of participants into any future observational studies. The fact that only five studies met the eligibility criteria, for what is currently considered the most prevalent type of scoliosis in adults, is testament to how difficult it is to focus research on this group.

In the cross-sectional study performed by Xu et al.[20], Cobb angle measurements were based on dual-energy x-ray absorptiometry (DEXA) scans. This type of scanning is typically performed with subjects in a supine position. It is well known that scoliotic curves reduce in magnitude in both the prone and supine positions. For this reason, the number of participants meeting the threshold for a diagnosis of scoliosis would have been reduced, deflating the associated prevalence rate in this particular study. All participants in the included studies were volunteers who attended some type of dedicated centre for the recording of the study outcomes. While some studies mentioned that participants were recruited irrespective of their level of mobility, the reality is that less mobile individuals with more severe spinal deformity would be less capable of travelling to a dedicated assessment centre. These individuals would likely be underrepresented in the study samples which could potentially lead to a reduction in the prevalence estimates calculated in this review.

The prevalence estimates for PDS presented in this review represent a pooling and refinement of the rates described in the current literature. However, a close inspection of the width of the confidence intervals highlights that further research is required in order to produce more accurate estimates of the prevalence of PDS and the influence of age and sex. Language restrictions imposed for this review may have resulted in an exclusion of important studies.

Eligible studies came from two regions: the Americas; and Asia. Only one study [15] involving 75 participants from North America was included in the review. The authors performed a subgroup analysis and have presented the prevalence estimates from the Asiatic region only. Inclusion of the Schwab et al. [15] study in the pooled prevalence does slightly inflate the prevalence estimates and the influence of age and sex. Further research is required to establish whether prevalence rates of PDS differ significantly between regions and if the pooled estimates detailed in this study can be generalized to other parts of the world.

\section{Conclusion}

Primary degenerative scoliosis is a highly prevalent condition affecting approximately $38 \%$ of the population. The condition is significantly more prevalent in females and is significantly more likely in adults aged $>60$ years. The majority of research in this area has come from the Asiatic region. Further research targeting this type of scoliosis is required to obtain more precise global prevalence estimates and to better understand the influence of age and sex.

Authors Contributions JM, CR, BS, AA and BTB designed the study. CR, BS, AA and BTB collected and collated the data. PLG performed the statistical analysis. JM, CR, BS, AA and BTB wrote the manuscript. All authors were involved with reviewing and editing the final manuscript.

Funding There was no funding received for this project.

\section{Compliance with ethical standards}

Conflict of interest There are no conflicts of interest to declare.

Open Access This article is licensed under a Creative Commons Attribution 4.0 International License, which permits use, sharing, adaptation, distribution and reproduction in any medium or format, as long as you give appropriate credit to the original author(s) and the source, provide a link to the Creative Commons licence, and indicate if changes were made. The images or other third party material in this article are included in the article's Creative Commons licence, unless indicated otherwise in a credit line to the material. If material is not included in the article's Creative Commons licence and your intended use is not permitted by statutory regulation or exceeds the permitted use, you will need to obtain permission directly from the copyright holder. To view a copy of this licence, visit http://creativecommons.org/licenses/by/4.0/. 


\section{Appendix}

\section{PubMed search strategy}

1. "Adult"

2. "Incidence" OR "Prevalence"

3. "Scoliosis" OR "De Novo Scoliosis" OR "De novo Degenerative Scoliosis" OR "Degenerative Scoliosis" OR "Adult Spinal Deformity" OR "Primary Degenerative Scoliosis" OR "Spinal Deformity” OR "Adult Scoliosis"

4. \#1 AND \#2 AND \#3

\section{References}

1. Stokes IA (1994) Three-dimensional terminology of spinal deformity. A report presented to the Scoliosis Research Society by the Scoliosis Research Society Working Group on 3-D terminology of spinal deformity. Spine 19(2):236-48

2. Bunnell WP (1986) The natural history of idiopathic scoliosis before skeletal maturity. Spine 11(8):773-776

3. Aebi M (2005) The adult scoliosis. Eur Spine J 14(10):925-948

4. Berven SH, Lowe T (2007) The Scoliosis Research Society classification for adult spinal deformity. Neurosurg Clin North Am 18(2):207-213

5. Wong E, Altaf F, Oh LJ, Gray RJ (2017) Adult degenerative lumbar scoliosis. Orthopedics 40(6):e930-e939

6. Weiss HR, Dallmayer R (2006) Brace treatment of spinal claudication in an adult with lumbar scoliosis-A case report. In: 6th Biennial meeting of the international research society of spinal deformities. Ghent

7. Buchowski JM (2009) Adult scoliosis: etiology and classification. Semin Spine Surg 21(1):2-6

8. Sun XY, Kong C, Zhang TT, Lu SB, Wang W, Sun SY et al (2019) Correlation between multifidus muscle atrophy, spinopelvic parameters, and severity of deformity in patients with adult degenerative scoliosis: the parallelogram effect of LMA on the diagonal through the apical vertebra. J Ortho Surg Res 14(1):276

9. Ferrero E, Skalli W, Lafage V, Maillot C, Carlier R, Feydy A, et al. (2019) Relationships between radiographic parameters and spinopelvic muscles in adult spinal deformity patients. European Spine Journal [Epub ahead of print]

10. Lowe T, Berven SH, Schwab FJ, Bridwell KH (2006) The SRS classification for adult spinal deformity: building on the King/Moe and Lenke classification systems. Spine 31(19):S119-S125

11. World Health Organisation (2018) Ageing and Health, https:// www.who.int/news-room/fact-sheets/detail/ageing-and-health. Accessed 23 Jan 2019

12. Good CR, Auerbach JD, O'Leary PT, Schuler TC (2011) Adult spine deformity. Curr Rev Musculoskelet Med 4(4):159-167

13. Torrie A, Adams MA (2015) Basic science of spinal degeneration. Surgery 33(6):233-237

14. Perennou D, Marcelli C, Herisson C, Simon L (1994) Adult lumbar scoliosis. Epidemiologic aspects in a low-back pain population. Spine 19(2):123-128
15. Schwab F, Dubey A, Gamez L, El Fegoun AB, Hwang K, Pagala M, Farcy JP (2005) Adult scoliosis: prevalence, SF-36, and nutritional parameters in an elderly volunteer population. Spine 30(9):1082-1085

16. Downes MJ, Brennan ML, Williams HC, Dean RS (2016) Development of a critical appraisal tool to assess the quality of crosssectional studies (AXIS). BMJ Open 6(12):e011458

17. Vandenbroucke JP, von Elm E, Altman DG, Gotzsche PC, Mulrow CD, Pocock SJ, Poole C, Schlesselman JJ, Egger M (2014) Strengthening the reporting of observational studies in epidemiology (STROBE): explanation and elaboration. Int J Surg 12(12):1500-1524

18. Higgins J, Altman DG, Sterne JAC (2011) Chapter 8: Assessing risk of bias in included studies. http://handbook-5-1.cochrane.org/. Accessed 4 Dec 2018

19. Hong JY, Suh SW, Modi HN, Hur CY, Yang JH, Song HR (2010) Correlation of pelvic orientation with adult scoliosis. J Spinal Disord Tech 23(7):461-466

20. Xu L, Sun X, Huang S, Zhu Z, Qiao J, Zhu F, Mao S, Ding Y, Qiu Y (2013) Degenerative lumbar scoliosis in Chinese Han population: prevalence and relationship to age, gender, bone mineral density, and body mass index. Eur Spine J 22(6):1326-1331

21. Jimbo S, Kobayashi T, Aono K, Atsuta Y, Matsuno T (2012) Epidemiology of degenerative lumbar scoliosis: a community-based cohort study. Spine 37(20):1763-1770

22. Hong JY, Suh SW, Modi HN, Hur CY, Song HR, Park JH (2010) The prevalence and radiological findings in 1347 elderly patients with scoliosis. J Bone Jt Surg Br 92(7):980-983

23. Pourhoseingholi MA, Vahedi M, Rahimzadeh M (2013) Sample size calculation in medical studies. Gastroenterol Hepatol Bed Bench 6(1):14-17

24. Gellhorn AC, Katz JN, Suri P (2013) Osteoarthritis of the spine: The facet joints. Nat Rev Rheumatol 9(4):216-224

25. Adams MA, Roughley PJ (2006) What is intervertebral disc degeneration, and what causes it? Spine 31(18):2151-2161

26. Adams MA, Dolan P (2012) Intervertebral disc degeneration: evidence for two distinct phenotypes. J Anat 221(6):497-506

27. Muraki S, Oka H, Akune T, Mabuchi A, En-Yo Y, Yoshida M et al (2009) Prevalence of radiographic lumbar spondylosis and its association with low back pain in elderly subjects of populationbased cohorts: the ROAD study. Ann Rheum Dis 68(9):1401

28. Cho HJ, Morey V, Kang JY, Kim KW, Kim TK (2015) Prevalence and risk factors of spine, shoulder, hand, hip, and knee osteoarthritis in community-dwelling Koreans older than age 65 years. Clin Orthop Relat Res 473(10):3307-3314

29. Ko S, Vaccaro AR, Lee S, Lee J, Chang H (2014) The prevalence of lumbar spine facet joint osteoarthritis and its association with low back pain in selected Korean populations. Clin Orthop Surg 6(4):385-391

30. Alshami AM (2015) Prevalence of spinal disorders and their relationships with age and gender. Saudi Med J 36(6):725-730

31. Wang Y, Peng R, Ma R (2013) Epidemiological investigation of osteoarthritis in middle-aged mongolian and senior residents of the inner mongolia autonomous region. Iran Red Crescent Med J 15(10):e8303

32. Eguchi Y, Toyoguchi T, Inage K, Fujimoto K, Orita S, Suzuki M (2018) Analysis of skeletal muscle mass in women over 40 with degenerative lumbar scoliosis. Eur Spine J 28(7):1618-1625

33. Tosi LL, Boyan BD, Boskey AL (2005) Does sex matter in musculoskeletal health? The influence of sex and gender on musculoskeletal health. J Bone Joint Surg Ser A 87(7):1631-1647

34. Mann CJ (2003) Observational research methods. Research design II: cohort, cross sectional, and case-control studies. Emerg Med J 20(1):54 
35. The Joanna Briggs Institute (2014) The Joanna Briggs Institute Reviewers' Manual 2014. The Systematic Review of Prevalence and Incidence Data. The Joanna Briggs Institute: The University of Adelaide, Adelaide, pp 1-37
Publisher's Note Springer Nature remains neutral with regard to jurisdictional claims in published maps and institutional affiliations. 